\title{
(2) \\ Tensión político-académica en la Universidad de Buenos Aires (1975-1983): el cambio de paradigma en la arqueología patagónica
}

\section{RMA}

Dossier

\author{
Susana Luco
}

Centro de Antropología Social, IDES, Buenos Aires, Argentina.

E-mail: susanaluco@yahoo.com.ar

\begin{abstract}
Resumen
La noción de sentido común de la historiografía antropológica a partir de la reinstauración democrática en 1983, ha sostenido que la producción de sus subdisciplinas ha quedado subordinada a las periódicas oscilaciones políticas sufridas por la Argentina desde la primera ruptura democrática de 1930.

El presente trabajo pone en cuestión tal aseveración, al menos en lo que concierne al segmento correspondiente a la arqueología patagónica practicada desde la Universidad de Buenos Aires, al postular que fue precisamente durante el gobierno militar autodenominado Proceso de Reorganización Nacional (PRN 1976-1983), en que se gestó el cambio de paradigma teórico desde la Escuela Difusionista Histórico-cultural centroeuropea a la Nueva Arqueología anglosajona (New Archaeology). Dicho cambio se originó en una primera instancia de quiebre metodológico que posibilitó una posterior ruptura teórica y que el mismo tuvo como artífice inicial al arqueólogo Carlos Aschero en tanto profesor regular de la licenciatura de Ciencias Antropológicas de la Facultad de Filosofía y Letras.
\end{abstract}

Palabras clave: cambio teórico; Universidad; Arqueología; Patagonia; Aschero

Political and Academic Strain in the University of Buenos Aires (1975-1983): Paradigmatic Change in the Argentine Archaeology of Patagonia.

\begin{abstract}
As common sense in Anthropological historiography has maintained since the restoration of democracy in 1983, the evaluation of anthropological production in each anthropological discipline has subordinated intellectual efforts to Argentine periodic political shifts ever since the first military coup took place in 1930.

This paper questions such notions regarding the Archaeology of Patagonia as it was taught and practiced at the School of Philosophy and Letters in the University of Buenos Aires. Conversely, this paper assumes that it was precisely during the latest military dictatorship, the self-labeled Process of National Reorganization (1976-1983), that a paradigmatic change took place from the Diffusionist Culture-History School of Central Europe, to the Anglo-Saxon New Archaeology. The inception of this shift, which was a methodological break led by archaeologist and professor Carlos Aschero, paved the way for the subsequent theoretical change. His regular courses and seminars for the licenciatura of Anthropological Sciences (Ciencias Antropológicas) provided the main settings of this creative process.
\end{abstract}

Keywords: theoretical change; University; Archaeology; Patagonia; Aschero.

La institucionalización universitaria de la licenciatura en Ciencias Antropológicas, ocurrida en 1958 en la Universidad de Buenos Aires' (en adelante UBA) no se alineó con los parámetros de "modernidad" y "desarrollo" que prevalecieron en la consolidación institucional universitaria de Sociología, Ciencias de la Educación y Psicología, creadas un año antes por la universidad reformista (Visacovsky, Guber, Gurevich 1997). En efecto, tras el derrocamiento político en septiembre de 1955 del segundo gobierno de Juan Domingo Perón, la

\footnotetext{
1 Esta actividad disciplinaria contaba con antecedentes académicos y universitarios desde fines del siglo XIX (Arenas 1989-1990, Figoli 1995, Podgorny 2004) Pero además en 1957 se había creado la licenciatura en la Universidad Nacional de la Plata en 1957, y la sede Rosario de la Universidad del Litoral.
}

autodenominada "Revolución Libertadora" que tomó a su cargo el Poder Ejecutivo Nacional (en adelante PEN), propició una "modernización" del Estado. En el estricto campo intelectual las nuevas medidas se orientaron a favorecer el desarrollo científico y tecnológico, a encontrar respuestas académicas a los problemas de la sociedad y a transformar el ámbito universitario. Esta modernización incluía la renovación de los cuerpos universitarios docentes y de la creación de nuevas carreras que se concretaron en 1957. La licenciatura en "Ciencias Antropológicas", nacida un año después, divergía de tal orientación debido a la permanencia de la mayor parte del cuerpo docente proveniente del período académico anterior enrolado en tradiciones centro-europeas como el difusionismo histórico-cultural en su variante vienesa 
que explicaba la pluralidad cultural como producto de la dispersión de bienes culturales desde ciertos centros al resto del mundo por difusión.

En el ámbito local los antecedentes de tal instauración teórica deben buscarse a comienzos de siglo XX con el arribo a la Argentina del etnólogo italiano José Imbelloni ${ }^{2}$ (1885-1967), impulsor de la Americanística y hombre fuerte de la Antropología durante la intervención peronista de la Universidad (1947-1955). Imbelloni encabezó una prolífica actividad profesional en universidades y museos nacionales, en clave difusionista. Más tarde, y tras la Segunda Guerra Mundial, hicieron su arribo otros exponentes del difusionismo quienes, como consecuencia de su compromiso con el nacional socialismo, se exiliaron en la Argentina merced al apoyo académico local de Imbelloni y, fundamentalmente, a la prédica de aquella escuela teórica en la Antropología que se practicaba en el país. El más destacado fue el prehistoriador vienés Oswald Menghin ${ }^{3}$ (1888-1973) formado en la Universidad de Viena, dónde se desempeñó como decano de la Facultad de Filosofía y Letras (1928-1930) y luego como su rector bajo la anexión alemana (1935). Menghin llegó a Buenos Aires en 1948 y fue incorporado inmediatamente a la Universidad. También hizo su arribo el romano Marcelo Bórmida (1925-1978) un joven graduado en antropología física y formado por Mario Sergi. Otros inmigrantes académicos se instalaron en el interior: el fisiólogo y raciólogo yugoslavo Branimiro Males (18971963) localizó su trabajo en la Universidad Nacional de Tucumán y el antropólogo húngaro Miguel de Ferdinandy en la Universidad de Cuyo. Menghin, quien permaneció en

\footnotetext{
2 Imbelloni orientó sus investigaciones hacia una disciplina que denominó Culturología centrando su trabajo, de marcado interés Americanista, en la reconstrucción de los patrimonios de los pueblos pero también en la definición y determinación de la relación existente entre grupos culturales y grupos raciales. Para ello apeló a su formación raciológica tan en boga en esos años adquirida en su país de origen. Su Epítome de Culturología (1936) y la traducción que el antropólogo español Salvador Canals Frau realizó de la Metodología Etnológica de Graebner, operaron como fuertes antecedentes para la instalación local del paradigma Histórico-cultural.

3 Oswald Frantz Ambrosius Menghin nació el 19 de abril de 1888 en Meran (Tirol austriaco). Estudió en la Universidad de Viena (1903) doctorándose en Filosofía en 1910 con especialización en Prehistoria. En 1913 fue profesor de Arqueología y Prehistoria, fundó la Weinner Präehistorische Zeitschrift, que se editó hasta 1943 y que luego reprodujo en la Argentina en la publicación "Acta Praehistórica" que dirigió en Argentina hasta su muerte. Fue profesor de la Universidad de El Cairo (1918/1945) y de la de Viena, así como Ministro de Educación de Austria como parte del partido católico y conservador que gobernó durante el Anschuluss de Austria en nombre del III Reich. Mantuvo una estrecha relación con el Instituto de Prehistoria y Arqueología de Barcelona mediante su revista Ampurias (1939). Desde su llegada al la Argentina se desempeñó como profesor de la UBA y desde la fundación de la Carrera de Ciencias Antropológicas en la UBA, (1958) estuvo al frente a la cátedra de "Prehistoria General y del Viejo Mundo" hasta su retiro a los 80 años. Asimismo enseñó Prehistoria en la Facultad de Ciencias Naturales y Museo de UNLP (1957). En ese mismo año fue rehabilitado como Profesor Universitario de Austria, y nombrado al año siguiente Miembro Honorario de la Sociedad de Antropología de Viena". Su prolífica obra incluye más de 900 escritos. Falleció en Chivilcoy, provincia de Buenos Aires, en 1973.
}

el ámbito universitario porteño desde la etapa peronista donde ingresó sin restricciones, renovando los honores con la dirigencia de la Universidad post-peronista, fue quien sistematizó la arqueología de Patagonia con la que se estructuró la enseñanza de esa subdisciplina en la UBA desde 1958 y hasta el cambio de plan de estudios de 1984.

Con la apertura democrática de 1983, punto de inflexión en la historia nacional argentina post-dictatorial, las historiografías de las ciencias sociales en general y de la antropología en particular emprendieron un balance y estado de situación de sus respectivos desarrollos disciplinares. A través de diversos artículos dieron cuenta del específico vínculo entre política universitaria y producción científica y las marcas que los regímenes de facto y su intervención en el ámbito universitario dejaron en la producción académica nacional. Algunos de esos artículos mostraron los bruscos cambios de dirección política a través de periodizaciones de la antropología en general (Bartolomé 1980, Figoli 1995, Madrazo 1982/1983) y de la antropología social en particular (Herrán 1987, Ratier-Ringuelet, 1997); otros artículos repasaron la particular constitución de la antropología social en la UBA (Visacovsky, Guber y Gurevich 1997, Guber y Visacovsky 1997/1998, Guber y Visacovsky 2000, Guber 2006, Guber 2008). Algunos, situados en subdiciplinas como la arqueología, segmentaron en etapas la historia de esa subdiciplina en la Argentina desde sus inicios (Fernández 1982, Orquera 1987) o bien se centraron en momentos críticos o esenciales de la misma (Núñez Regueiro 1971, González 1985, Boschín-Llamazares 1986, Boschín 1991/1992, González 1991/1992, Politis 1988, 1992, Haber 1994, Podgorny 1999, Politis 2004, Gollán y Kohl 2002, Tarragó 2006) (LuCo 2009: 3).

La mayor parte de las historias y periodizaciones de la antropología han priorizado una noción de sentido común político según la cual las producciones, selecciones y adscripciones teórico-metodológicas que primaron en la antropología y sus subdiciplinas, debían comprenderse en razón directamente proporcional a la realidad social y política que las acompañó (Guber 2007). En tanto que otros han resaltado la particular modalidad que establecía una ecuación según la cual los años de regímenes no democráticos eran sinónimo de estancamiento o retroceso en la producción académica, mientras los de regímenes democráticos quedaban asociados al avance y la innovación teórica disciplinar. En el específico ámbito de la antropología porteña esta interpretación se tradujo también a subdisciplinas completas, ya que los procesos renovadores habrían promovido a la Antropología Social, proscripta por la academia oficial en períodos dictatoriales, más generosos con la arqueología y la etnología.

En las páginas siguientes expondré un caso que contradice 
tal concepción pues si bien la arqueología practicada en la licenciatura de Ciencias Antropológicas de la Universidad de Buenos Aires (UBA) en el decenio 1970/1980 sufrió restricciones institucionales y prohibiciones que delimitaron la práctica de campo y la discusión teórica, fue precisamente durante el autodenominado Proceso de Reorganización Nacional ${ }^{4}$ (1976-1983, en adelante PRN) que tuvo lugar la introducción, implantación, enseñanza y adscripción a un nuevo paradigma que desafiaba profundamente al paradigma oficial. En esta investigación, que procura contribuir a una etnografía de los cambios teóricos de las comunidades científicas, intento señalar la complejidad que revistió un proceso que no fue operado desde el exterior ni como la mera importación de nuevos modelos, sino más bien como un encadenamiento de selecciones, adecuaciones y reformulaciones desde dentro de la unidad institucional que administraba la práctica y enseñanza de la subdiciplina arqueológica porteña ${ }^{5}$. Así pues, para recuperar la especificidad del tema en estudio a través de las pocas líneas que impone un artículo de estas características, me es necesario precisar que el mismo describe y analiza, mediante una narrativa etnográfica, el discurso de los actores sociales de esta investigación: los arqueólogos patagónicos que intervinieron en dicho proceso de cambio desde la Universidad de Buenos Aires. Es desde esa perspectiva y sólo desde ella, que he narrado e interpretado cómo fue ese proceso, y he caracterizado a quiénes lo llevaron a cabo en el estrecho marco de la universidad porteña bajo las normas y restricciones de la dictadura militar, destacando que, pese a dicho contexto, sus protagonistas (y no yo misma) conciben el cambio como un verdadero avance en el desarrollo de la arqueología nacional. Dicho proceso cobró, inicialmente, características de índole metodológica para luego dar paso a un efectivo cambio teórico. Con ese objetivo caracterizo además a quien, según sus contemporáneos, fue el agente inicial del cambio en su faz metodológica, el joven arqueólogo patagónico Carlos Alberto Aschero.

\section{La tradición histórico-cultural como organizadora del campo de la prehistoria de Patagonia}

Desde la creación de la licenciatura porteña en 1958 y el comienzo de su dictado en 1959 fue el prehistoriador austriaco Oswald Menghin, Ilegado al país en 1948, el

\footnotetext{
4 El golpe militar de marzo de 1976, que instaló en el poder político a las Fuerzas Armadas inició el PRN y estableció una práctica represiva de las distintas "organizaciones peronistas y marxistas guerrilleras que habían interpretado las protestas masivas de trabajadores y estudiantes de 1969 como un presagio de la revolución" (Robben 2005:10), extendiendo ese mismo criterio a la sociedad argentina toda. En consecuencia, las actividades comunitarias fueron severamente restringidas, a través de una estricta supervisión que recayó principalmente sobre aquellos espacios en los cuales se sospechaba la posible articulación y fomento de actividades de concientización y militancia políticas, por entonces calificadas de "subversivas" (Visacovsky 2002).

5 Para conocer las reflexiones acerca de la posición subordinada de la praxis arqueológica en el orden neo-colonial ver Politis 2004, así como las discusiones que este trabajo promovió en la Revista Suramericana 2 (2) de julio de 2006.
}

responsable principal de las exploraciones arqueológicas realizadas en las regiones de Pampa y Patagonia. Su conducción teórico-institucional cabía dentro de los preceptos de la Escuela Etnológica Europea, fundada por dos etnólogos de habla germana, Wilhelm Schmidt (1868-1911) y Franz Graebner (1877-1925) ${ }^{7}$. Para ellos los "ciclos culturales" explicaban la distribución territorial de una cultura arcaica única y original de cuyos primigenios rasgos constitutivos se habrían generado luego los demás desarrollos culturales mediante un proceso de dispersión geográfica. Etnólogos y arqueólogos se daban a la tarea de rastrear y reconstruir esos ciclos, a la manera de las isotermas (Figoli 1995: 297, Kohl \& Pérez Gollán 2002).

En la Argentina el eco de la controversia AmeghinoHrdlicka sobre el origen del hombre americano (1910) conllevó el abandono de las investigaciones científicas sobre "el hombre antiguo" de la región pampeana aún cuando se siguió investigando sobre otras problemáticas del área a través de exploraciones como las encabezadas entre muchos otros por Lothrop, De Aparicio, Torres, etc; en tanto la vasta Patagonia carecía de investigaciones consistentes a excepción de las realizadas por Félix Outes (1878-1939) en $1905^{8}$ y más tarde por las de Junius Bird (1936). En los primeros años de la década de 1950 Menghin reavivó la problemática del poblamiento, trasplantando los ciclos propuestos por la Escuela Histórico-Cultural austro-alemana y proponiendo una sistematización en base a períodos y fases de acuerdo al concepto de oleadas culturales aplicado a Pampa y Patagonia9 (Imbelloni 1947, Menghin 1952). Menghin

\footnotetext{
6 Nació en Horde, Alemania. Inició la Escuela difusionista austriaca. Desarrolló su tarea etnológica en su variante regional difusionista como integrante de la Sociedad de la Orden de misioneros del verbo divino de Austria. Si bien estuvo influenciado por el antropólogo norteamericano Franz Boas, su maestro fue Graebner del que recibió la concepción de la Kulturkreise. Fundó la publicación "Anthropos" (1906), donde se presentaban los hallazgos etnográficos producto de sus viajes por Nueva Guinea y Togo.

7 Nació en Berlín. En 1904 presentó los Kulturkreise de África y Oceanía ante la Sociedad Antropológica de Berlín y luego volcó esta teoría en un manual, Methode Ethnologie Kulturmorphologie. Se abocó junto a B. Ackermann, otro representante de ésta Escuela, a clasificar las colecciones de los mares del sur. Como promotor de la investigación de su época, interpretó la historia de Oceanía a partir de la difusión geográfica de agrupamientos de rasgos culturales que volcó en un mapeo de su dispersión para visualizar las recurrencias culturales que agrupó en "complejos culturales".

8. Nació en Buenos Aires en 1878. Fue antropólogo, arqueólogo y lingüista. Organizó el Museo Etnográfico de la Facultad de Filosofía y Letras de Buenos Aires. Fue parte de la segunda generación de naturalistas argentinos, continuadores de Ameghino, Moreno, Ambrosetti y Holmberg. Entre 1903, y 1911 integró la sección arqueológica del Museo Nacional de Historia Natural. Dictó clases en la Facultad de Ciencias Naturales de La Plata, y dirigió los Anales del Museo donde dio a conocer trabajos de su especialización. Enseñó Geografía Humana en la UBA (1914) y organizó el Instituto de Investigaciones Geográficas. En 1930, reemplazó a Lehmann-Nitsche en la cátedra de Antropología, y dirigió el ME hasta 1938. Fundó la Sociedad Argentina de Antropología. Falleció en Bs. As el 8 de septiembre de 1939.

9 En Patagonia realizó dos campañas. La primera desde enero a abril de 1951 y la segunda de enero a fines de marzo de 1952. Sus resultados conformaron tres artículos "Las pinturas rupestres de la
} 
enfatizó los conceptos de contacto cultural e intercambio para explicar el cambio cultural en ambas regiones, aunque Patagonia tenía un interés especial por su condición de finis terrae ${ }^{10} \mathrm{y}$, por lo tanto, de zona de "arrinconamiento" de los pueblos más antiguos llegados a América. Con su joven asistente, Bórmida y José Imbelloni, Menghin conformó un cerrado equipo en el Instituto de Antropología de la Facultad de Filosofía y Letras de la UBA dónde se instituyó la autodenominada "Escuela de Buenos Aires".

El mapa prehistórico trazado por Menghin correspondía a una configuración de índole ecuménico-especulativa, con la que proyectaba documentar las distintas tradiciones del período arqueológico denominado Paleolítico Inferior (continuando la nomenclatura europea), y los contactos significativos entre Viejo y Nuevo Mundo. De acuerdo a dicha configuración, la Patagonia habría sido ocupada por dos tradiciones culturales que coexistieron hasta nuestra era: la Epiprotolítica (10.000 - 7.000 a.p.) y la Epimiolítica (8.000 a.p) (Menghin, 1952, 1957b). En la terminología del prehistoriador vienés los prefijos "Epi" y "Mio" aludían al epifenómeno que suponía esta suerte de desfasaje cultural que ambas tradiciones guardaban con relación al modelo de periodización empleada por la prehistoria europea. A su vez ambas tradiciones contenían manifestaciones culturales locales que Menghin Ilamó "Industrias", denominadas según el nombre geográfico donde se hubieran descubierto. En todos los casos subyacía la noción de continuidad en la ocupación de estos cazadores que habrían llegado y ocupado el territorio patagónico en sucesivas oleadas desde el oeste por los Andes, y desde el este por la Amazonia. Así, una tradición representaba a cazadores portadores de un conjunto de artefactos poco especializados en sus traslados por la meseta patagónica, de tipo "más bien 'rudimentario, es decir, (...) se trata casi exclusivamente de un instrumental manufacturado de lascas $^{11}$ con o sin retoque" (Menghin 1952b:27). La otra tradición estaba asociada a artefactos atribuidos a cazadores superiores, un conjunto "especializado, para afrontar la caza de guanacos y algunos otros recursos de fauna que completaban su dieta, contando con puntas de dardo pedunculadas tempranamente, pequeños raspadores y raederas" (Menghin 1952b:34). Para el prehistoriador existía evidencia material que revelaba que los cazadores

Patagonia" "Fundamentos cronológicos" y "Estilos de arte rupestre" publicados en Runa y Acta Praehistórica, órganos de difusión de los difusionistas de la UBA.

10 Esta argumentación fue sostenida también por su discípula Amalia Sanguinetti, quien ha señalado "No cabe duda que si en un lugar, las culturas arqueológicas y etnográficas (...) se dan en una línea ininterrumpida, ese lugar es la Patagonia. En parte, por haber llegado a ella tardíamente las más antiguas corrientes de poblamiento de América donde se han conservado, (...) hasta etapas muy recientes" (Sanguinetti 1972: 8).

11 Lámina obtenida percutiendo con un instrumento lítico, una porción de piedra mayor y a partir de la cual se manufactura una herramienta lítica como raederas o raspadores que se caracterizan por tener borde activos para su uso cortante de carne y cuero. inferiores y los cazadores superiores habían convivido y compartido los mismos recursos y territorios; "(existen) residuos (materiales) de culturas de los cazadores inferiores que vivieron contemporáneamente con los cazadores superiores que habían inmigrado a América o a la misma Patagonia" (Menghin 1952 b: 41, mis paréntesis).

Este esquema conceptual, con el que se impartió la formación de grado de sucesivas generaciones de arqueólogos de Patagonia desde los 1960 en adelante, establecía una directa correspondencia entre secuencias arqueológicas y secuencias culturales. En esa misma década, y con el fin de establecer también una interpretación basándose en correlaciones culturales directas entre los grupos humanos del paleolítico y su "equipamiento material", se incorporó a la enseñanza "oficial" de las cátedras porteñas el recurso metodológicoarqueológico de la tipología ${ }^{12}$ que el prehistoriador francés François Bordes ${ }^{13}$ (1919-1981) había elaborado sobre la base de los materiales de yacimientos del Paleolítico Inferior y Medio del viejo mundo: Pech-de l'Azé, Combe-Grenal y Corbiac.

A mediados de la misma década el joven Carlos Alberto Aschero (n.1946), ingresaba a la licenciatura de Ciencias Antropológicas de la UBA en el marco del primer plan de estudios de la carrera ${ }^{14}$, y que finalizaría en 1974. Algunos de sus docentes fueron el ya citado Bórmida, Ciro R. Lafón (1923-2006) y Luis A. Orquera. Allí adquirió sus primeras nociones del trabajo de gabinete, facilitadas por su temprano interés por la tipología lítica y gracias al cual obtuvo el cargo de Técnico de laboratorio ${ }^{15}$. Pudo así acceder a las colecciones del Museo Etnográfico dependiente de la UBA. De esa época son también sus primeras experiencias de campo, con el acompañamiento personal y profesional de Carlos Gradin, topógrafo y técnico de Menghin en el CONICET ${ }^{16}$, y de su compañera

\footnotetext{
12 Dicho recurso extendido desde 1930 fue utilizado por las escuelas académicas de las arqueologías estadounidense, francesa y alemana para clasificar, ordenar y analizar los artefactos líticos y así interpretar el uso que se les había acordado en el pasado más remoto.

13 Estudió en Toulouse, en Burdeos y en París doctorándose en ciencias naturales. Fue parte del CNRS entre 1945 y 1955 y titular de las materias de Geología del Cuaternario y Prehistoria en la Facultad de Ciencias de Burdeos (1956), dónde fundó el Instituto del Cuaternario (hoy Instituto de Prehistoria y Geología del Cuaternario del CNRS). Su mayor contribución se relaciona con las industrias líticas del Paleolítico inferior y medio y con una metodología empírica conocida como el «Método de Bordes».

14 El Plan 1958, según Resolución CA 50/59, contenía tres especializaciones.(Etnología, Folklore y Prehistoria) y con excepción de algunas pequeñas variaciones de contenido, mantuvo su vigencia entre 1959 (comienzo de dictado efectivo de la Licenciatura) y 1973. El plan consistía en el cursado de 26 materias y un trabajo final ó cursillo en cualquiera de las tres orientaciones completado con su respectivo informe. (Guber 2007).

15 Aschero ejerce el cargo entre diciembre de 1969 y Mayo de 1974, con una localización laboral en el Museo Etnográfico.

16 Mediante el Decreto 1251/58 (B.O: 19/02/59) se creó el Consejo
} 
de estudios Ana Margarita Aguerre. Como docente auxiliar fue en primer lugar ayudante de trabajos prácticos ad honorem y luego rentado, de las asignaturas "Prehistoria General y del Viejo Mundo" y "Prehistoria y Arqueología Americana I". Ambos cursos correspondían al segundo y tercer año de la carrera y su titular era Amalia Sanguinetti de Bórmida.

En este cargo Aschero lograba una comunicación inusual con los alumnos con quienes compartía los nuevos conocimientos que producía basándose en la propia iniciativa y a la experiencia de su trabajo de gabinete y sus campañas. Tras obtener su título de grado (1974) y ya como becario de iniciación del CONICET, presentó su primer informe, un trabajo de sistematización de la tipología existente aplicada a materiales específicos de la región patagónica. Este Ensayo para una clasificación morfológica de artefactos líticos aplicada a estudios tipológicos comparativos ${ }^{17}$ alcanzó una gran trascendencia entre los colegas interesados en contar con una elaboración integral sobre el material lítico. "La tipología de Carlos", como se conoció informalmente a este reporte, fue el puntapié inicial del proceso que analizo aquí.

\section{La consagración de nuevos espacios}

A pesar de que para 1975 la práctica arqueológica de la UBA estaba todavía enmarcada por la perspectiva difusionista y las posibilidades de adquirir las destrezas profesionales requeridas para un principiante no eran muchas, Aschero ya había tomado contacto con buena parte de ellas. Su experiencia de campo en Patagonia y el NOA, aún con una escasa antigüedad en la carrera y en el métier, le había dado un mayor conocimiento de las prácticas corrientes en la arqueología patagónica menghiniana con las que fue configurando sus propias ideas. El acceso a una bibliografía constituida por los trabajos de varios de los arqueólogos de mayor notoriedad que por entonces moldeaban el cambio de ideas en las academias arqueológicas de Francia, Inglaterra y Estados Unidos coadyuvó a que este novel arqueólogo iniciara un lento despegue de su herencia histórico-cultural. Su práctica profesional de campo coincidía con la perspectiva empírica que asimiló de la academia francesa y que formaba parte de la enseñanza "oficial" de la arqueología porteña. Pero las contradicciones, dicotomías y fracturas teórico-metodológicas del esquema defendido por Menghin para explicar la temprana presencia humana en Patagonia como la dicotomía entre "importación"

Nacional de Actividades Científicas y Tecnológicas (CONICET), como ente autárquico con la misión de promover, coordinar y orientar las investigaciones en el plano de las ciencias puras y aplicadas. Con su creación se suprimió la Dirección Nacional de Investigaciones Científicas y Técnicas. En 1961, se creó la carrera del investigador, y en 1967, la de técnico.

17 Aschero, Carlos A. 1975. Ensayo para una clasificación morfológica de artefactos líticos aplicada a estudios tipológicos comparativos. 162 páginas. Inédito. Buenos Aires. y "desarrollo local", comenzaron a dejar al descubierto la posibilidad de indagar si las inferencias basadas en la difusión permitían dar cuenta del verdadero modo de vida de los cazadores patagónicos. Preguntas tales como de qué modo y por qué un grupo humano no había producido una respuesta cultural acorde a su medio, comenzaron a ponerse en cuestión entre algunos miembros de las cohortes que cursaban su formación de grado en arqueología en la licenciatura de la UBA en los '70. Este cuestionamiento requirió no sólo de tiempo, sino también del desarrollo de métodos y de nuevas nociones para la clasificación de los materiales líticos.

\section{La "Sala de las columnas"}

Durante el segundo cuatrimestre de 1976 y el primero de 1977, Bórmida, director de la licenciatura, aprobó el dictado de tres seminarios de arqueología aprovechando la modalidad curricular de la Facultad de destinar la etapa de especialización de la carrera de grado a la profundización de ciertos temas de investigación. Con el bagaje de su especialización en la arqueología de la región patagónica y del noroeste argentino, y con su interés por el arte rupestre, a poco de obtener su graduación Aschero logró la aprobación para el dictado de dos de ellos. El primero se centró en "Arte rupestre en el territorio argentino" y se llevó a cabo sobre la base del material obtenido en sus investigaciones con Gradin y Aguerre en la "Cueva de las Manos" (Prov. de Santa Cruz) y con las manifestaciones de los sitios Inca Cueva y Huachichocana ${ }^{18}$ del norte argentino. Esta temática había comenzado a imponerse en la agenda de investigación nacional al demostrar que en dicha región se distinguían complejos niveles de ocupación humana que precedieron a las sociedades agroalfareras. El segundo seminario se abocaba exclusivamente a Patagonia ${ }^{19}$. Probablemente debido a la poca oferta en las diferentes áreas de estudio, una considerable cantidad de alumnos de Etnología y Folklore, las otras dos especializaciones de la licenciatura, decidió inscribirse en dicho Seminario por lo que Aschero resolvió añadir a su programa inicial algunos temas extraarqueológicos.

Interesado en poner a prueba la eficacia de su Tipología y volcándose a la obtención de una caracterización morfológica de la "Industria Tehuelchense" o "Patagoniense" definida de modo general por Menghin (1952, 1957a, 1957b) y luego por Bórmida (1964)

\footnotetext{
18 Inca-Cueva 4 y 7 y Huachichocana son sitios arqueológicos emplazados en la Puna septentrional jujeña que demuestran la ocupación temprana de esta región por parte de bandas de cazadores de camélidos provistos de proyectiles arrojadizos con los que desplegaron diversas técnicas de caza y que habitaron temporariamente, como los cazadores patagónicos, cuevas y aleros emplazados a 3200 mts de altura. También aquí Aschero estudió el arte de estos grupos.

19 El dictado del tercer Seminario estuvo a cargo de la arqueóloga Marta Pastore, adjunta de Sanguinetti, y en el se reseñaba el desarrollo cultural de Nordpatagonia desde el esquema histórico-cultural ortodoxo.
} 
con colecciones inéditas de las sucesivas campañas de Menghin, Gradin y Aschero (Pérez de Micou 2006:2), Aschero dictó un Seminario sobre Patagonia que sólo tuvo un cuatrimestre de duración y que, sin embargo, ha permanecido en la memoria de quienes concluían su formación de grado entre 1976 y 1977. Así, organizó a aquellos inscriptos interesados en trabajar con él en grupos de trabajo de manera que cada alumno se hiciera cargo, como operador técnico, de someter una a una las piezas arqueológicas de la colección adjudicada a su grupo, a las instancias de análisis planteadas por su Tipología. Por motivos de orden práctico y probablemente porque esta tarea no estaba prevista en las actividades de un seminario tradicional; en lugar de un aula Aschero prefirió reunir a los alumnos en un amplio recodo conocido como la "Sala de las Columnas", en la planta baja de un edificio de la Facultad en el micro-centro porteño. La "Sala de las Columnas" debía su nombre a unas inmensas y gastadas columnas desprovistas de todo estilo que dominaban una vasta y oscura zona del ala derecha del hall de ingreso al viejo edificio que otrora albergara al Banco Hipotecario Nacional. Al parecer, la falta de límites precisos de este espacio sin destino que hacía las veces de paso lateral inhabilitado que conducía a ninguna parte, lo terminó de configurar como una amplia área disponible. El trabajo técnico de estudio de las piezas se hacía en torno a una única y larga tabla de madera de aproximadamente diez metros de largo. Cada equipo tenía a su cargo la aplicación de las premisas de la Tipología y el uso de referencias bibliográficas. El producto final del Seminario fueron siete trabajos, uno por cada sitio. ${ }^{20}$

Del mismo modo que la "Sala de las Columnas" los situaba fuera del recinto de las aulas, la actividad y los propósitos del Seminario se ubicaban fuera de las actividades curriculares y de la modalidad investigativa acostumbrada del Instituto de Antropología de la Facultad donde habitualmente se desarrollaban esa clase de tareas. Porque más allá de la peculiaridad de la tarea encomendada por Aschero a sus estudiantes, "ser muchos y estar todos juntos" en un espacio distinto y de características liminares como el del Seminario le confería al docente responsable y a la propia tarea, un sentido fuertemente simbólico en el contexto de control y vigilancia a cargo del PRN y de la "Escuela de Buenos Aires". Por iniciativa de Aschero los cursantes del Seminario completaron su trabajo con el acceso a la bibliografía ${ }^{21}$ y a las libretas de campo correspondientes

\footnotetext{
20 Un análisis tipológico y técnico-morfológico de siete sitios del complejo patagoniense (Ms).Aschero, Carlos, Bellelli, Cristina, Fernández Lannot, Carmen, Fisher, Alfredo, Fontanella, María V, Gómez Otero Julieta, Pérez de Micou, Cecilia.

21 Laguna Azul, con la referencia bibliográfica de Menghin y Cecilia Pérez de Micou como operadora de tipología, Chacra Briones, con referencia bibliográfica anónima y $\mathrm{M}^{\mathrm{a}}$ Victoria Fontanella y Cristina Bellelli como operadoras de tipología, Piedra Calada con la referencia bibliográfica de Menghin y Gradin y Alfredo Fisher como el operador de la tipología, Bahía Solano con la referencia bibliográfica de Menghin
}

que, en su gran mayoría, pertenecían a campañas realizadas por Menghin no sólo retirado de la Facultad desde 1969, sino para ese entonces, ya fallecido. En este material inédito los alumnos encontraban las condiciones en que se habían realizado los hallazgos, accediendo, por ejemplo, a las plantas de alguna de aquellas excavaciones.

La innovadora experiencia de la "Sala de las Columnas" con su ubicación física intersticial y transicional, es resignificada como una manifestación metafórica del primer hito de una suerte de pasaje simbólico que Aschero recorrió junto a aquellos alumnos interesados en "llegar a ser arqueólogos". El trabajo sobre los siete sitios fue presentado como de autoría conjunta, al V Congreso Nacional de Arqueología en la provincia cuyana de San Juan en 1978.

Sin embargo, pese a su éxito, tanto la Tipología como el trabajo de los 7 sitios que originó, quedaron inéditos durante los siguientes treinta años. La publicación de los 7 sitios se efectivizó recién en 2007, como Un análisis tipológico y técnico-morfológico de siete sitios del Complejo Patagoniense (Ms). Su editora y actual titular de la asignatura "Ergología y Tecnología" Cecilia Pérez, señala en la introducción que "para la mayoría de los autores era su primer trabajo. Nunca vio la luz. Sin embargo circuló fotocopiado y fue citado en muchas oportunidades. Su aparición (...) intenta cubrir aquella falta y homenajear a su mentor, el Profesor Carlos Aschero"22. Entre tanto la Tipología jamás se publicó.

\section{"La tipología de Carlos"}

Aquel informe presentado por Aschero al CONICET se apoyaba en un ya probado recurso metodológico en el mundo arqueológico, consistente en una instancia taxonómica o clasificatoria de los materiales líticos locales. Su eficacia en el ámbito académico internacional resultaba de la búsqueda con la que los prehistoriadores, ya en la década de 1930, intentaron hacer propuestas que fueran más allá de la mera descripción y rotulación de los materiales de excavación, características de las primeras épocas de la práctica disciplinar. Esta nueva herramienta metodológica permitió explicar características culturales recurrentes, a través del estudio de los utensilios líticos. A través de una detallada jerarquía clasificatoria, la tipología daba acceso a un cierto tipo de explicación vinculado al cerrado vínculo entre la manufactura del objeto y la conducta cultural de su hacedor. El gran auge académico alcanzado por esta opción metodológica entre los estudiosos franceses, italianos, españoles y anglosajones

(1952) y Mario Pitluk como operador, Cabo Blanco con referencia bibliográfica de Outes (1905), Vignati (1930) y Molina (1967) y Carmen Fernández Lannot como operadora de tipología, Colhué Huapi con referencias bibliográficas de Menghin (1952) y Liliana Raggio como operadora de tipología y Tapera 2, con referencia bibliográfica de Gradin (1973) y Aschero (1975) y Julieta Gómez Otero como operadora de tipología.

${ }^{22}$ ver Pérez de Micou, C. 2006. 
como procedimiento para estudiar las sociedades del pasado, convergió a fines de los '50 en la propuesta tipológica del antes citado Bordes que alcanzó una amplia acogida y adopción internacional.

En el panorama arqueológico nacional deben mencionarse varias iniciativas "tipológicas" que se orientaban en una línea diferente de la aplicación clasificatoria ortodoxa de las exploraciones histórico-culturales para los escenarios territoriales de Pampa y Patagonia. Los trabajos de Austral y de Guillermo Madrazo (1927-2004) en el área pampeana procuraban dar un vuelco al planteo de Menghin y Bórmida. Austral, socializado como históricocultural, incorporó un giro para el análisis de los materiales de tres industrias líticas del litoral bonaerense que ya había trabajado Bórmida: el "Palomarense" en el sitio el "Palomar" en la región de Pampa, 70 Km. al sur de Bahía Blanca (Prov. de Buenos Aires), el "Puntarrubiense" y el "Jabaliense". Por su parte Madrazo fue más allá al incorporar la mirada ecológica en un intento por interpretar la secuencia de ocupación humana de la región durante el Holoceno tardío. Y a ellos deben sumarse las propuestas de Rita Ceballos y de Orquera quien había iniciado un desplazamiento hacia una propuesta tipológica propia a la luz de los hallazgos en los sitios de "Lancha Packewaia" y "Túnel" sobre el Canal de Beagle en la Prov. de Tierra del Fuego (Luco 2009: 45).

En este contexto la "Tipología de Carlos" surgió bajo las influencias del trabajo de Bordes pero también de otros autores ${ }^{23}$, en respuesta a las propias preguntas de campo y a las observaciones morfológicas nacidas en el gabinete del Museo Etnográfico. En su introducción Aschero señalaba que la misma era "el resultado de una síntesis de diversos criterios descriptivos aplicables a materiales líticos de morfología paleolítica elaborados por talla o retoque" (Aschero 1975:1) y que en ese sentido "cumple con la necesidad de ordenar los pasos y las categorías del análisis tipológico" (Ibíd. 1975:1). Destacaba que lo animaba la idea de "lograr una mayor especificación en la determinación de 'tipos' dentro de los conjuntos líticos que se atribuían a la llamada industria "Patagoniense" o "Tehuelchense" de la Patagonia argentina". Con ella proponía también "intensificar los aspectos descriptivos o documentales de los hallazgos, a fin de que sirvieran a distintos investigadores en distintas áreas" y "para que en el futuro se especifique la nomenclatura tipológica y se presenten listas tipológicas que puedan ser estadísticamente representadas" (Aschero 1975:2). Ya posicionado en el escenario arqueológico de Patagonia, Aschero se dispuso a repasar a partir de su clasificación analítica de los artefactos líticos los puntos de búsqueda que animaban su revisión de la caracterización históricocultural del Patagoniense definido por Menghin. Pero esta sistematización analítica, eminentemente técnica

\footnotetext{
23 Ante nuestra consulta acerca de las influencias para la confección de su tipología Aschero enumeró no sólo a Bordes, Laming-Emperaire, Leroi-Gourhan sino también a Wormington, Irwin y Bagolini.
}

y sin pretensiones de transitar por carriles teóricos, no sólo presentaba un repertorio discreto y detallado de las opciones de análisis técnico-tipológicos y morfológicos pasibles de ser utilizadas en futuras investigaciones según un lenguaje común y uniforme; sino que exteriorizaba también signos de divergencia con la matriz difusionista en la que se había formado. En efecto, un ejemplo de ello es que Aschero remarcaba en su presentación la necesidad de hacer "observaciones" morfológicas de conjuntos líticos reales y no de codificaciones "posibles", práctica ésta distintiva de los difusionistas ortodoxos porteños.

Si bien se proponía poner orden en las concepciones tipológicas foráneas dando la posibilidad de organizar y sistematizar con ellas los materiales locales, la tipología vernácula de Aschero logró encarnar, entre los arqueólogos líticos nacionales, mucho más que un material de síntesis listo para usar. Su fuerte efecto en el resto de sus colegas residió en el hecho de reconocer que cualquier adopción mecánica, esencialista o normativista traía aparejados serios problemas de aplicación al dificultar su "adaptación" al ámbito local. Con su tipología, Aschero proponía "revisar y replantear la utilidad de las descripciones tipológicas en el marco del análisis cultural prehistórico, tratando de señalar su papel y su limitación para acceder a la "reconstrucción cultural" (Aschero 1975:2). Su aplicación comenzó a dejar al descubierto las fisuras de la arqueología preexistente que se caracterizaba por una transposición del modelo ecuménico del Paleolítico europeo y que conservaba la vigencia de las categorías reconstructivas del difusionismo europeo.

En su Seminario sobre Patagonia Aschero puso a prueba la eficacia metodológica de su topología al tiempo que planteó una suerte de "revisionismo" de los materiales canónicos del difusionismo patagónico, logrando no sólo un lento desplazamiento de las estructuras de análisis preexistentes sino también una temprana afirmación en el ámbito académico nacional gracias a la aceptación de buena parte de sus colegas líticos. A semejanza de la modalidad articuladora que ocupó su tipología en la escena arqueológica nacional definida por Orquera como "una minuciosa adaptación local influenciada por la tipología de Bordes, cuya unidad analítica era el 'artefacto', y por la del sistema americano cuya unidad de análisis era el 'rasgo' (...) de gran utilidad primero para clasificar los materiales existentes y más tarde para poder interpretarlos"24 (Entrevista a Orquera 11/10/2007),

\footnotetext{
24 Como producto de la Primera Convención Nacional de Antropología reunida en Carlos Paz del 24 al 29 de mayo de 1964 con el propósito de unificar criterios terminológicos que la arqueología había puesto en uso, se estableció una diferencia para el uso profesional entre "útil" y "artefacto": "útil es todo objeto lítico empleado por un ser humano (...) en estado natural, es decir (...) sin la alteración intencional del mismo para adaptarlo como por ejemplo un guijarro natural o un canto rodado. en tanto, "artefacto" refería a todo objeto que ha sido producido por el ser humano. producido indica una transformación intencional, una alteración de las características naturales, es decir es resultante de una actividad humana activa, por ejemplo una punta de
} 
Aschero fue, él mismo, un articulador de las relaciones personales del medio académico porteño de la década de 1970. Si bien su socialización e inserción institucional lo ligaban a la orientación difusionista, su experiencia investigativa y docente lo acercaba a una nueva forma de hacer arqueología. Este nuevo rumbo de la arqueología patagónica se expresaba en función de una necesidad, no satisfecha hasta entonces, de establecer la congruencia entre "interpretación" y "dato" producida por la interpretación histórico-cultural ortodoxa. Cabe pues aquí preguntarse cómo daba cuenta Aschero de las fisuras del sistema teórico de la arqueología preexistente en el que se había socializado.

Como ya hemos dicho, la nutrida experiencia de campo y de gabinete acumulada en pocos años, sumados al acceso a una bibliografía ecléctica generada por varios de los arqueólogos que entonces moldeaban el cambio de las ideas en las academias de Francia, Inglaterra y Estados Unidos, contribuyeron a que Aschero influenciado por Bordes, pero también por los prehistoriadores galos Anette Laming-Emperaire (1917-1977) y André LeroiGourhan. ${ }^{25}$ (1911-1986), iniciara un lento despegue de su legado histórico-cultural. Su práctica de campo coincidía con la perspectiva empírica que asimiló de la academia francesa pero enriquecida además por las innovaciones introducidas por Leroi-Gourhan y su meticulosa propuesta de recolección del material lítico como parte del desarrollo de nuevas técnicas orientadas a optimizar los distintos procedimientos metodológicos del trabajo de campo.

Si bien en la producción académica de sus primeros años de investigación Aschero estuvo dispuesto a adscribir sus hallazgos como "tradiciones culturales", "entidades culturales" e "industrias" propias de la corriente difusionista de la "Escuela de Buenos Aires" (Aschero 1975a; Gradin, Aschero, Aguerre 1976; Gradin, Aschero, 1978; Gradin, Aschero, Aguerre, 1979; Aschero, Bellelli, Fontanella, 1980; Aschero 1981a y b; Aschero 1981/1982; Aschero 1982; Aschero 1983; Aschero 1983/85; Alonso, Gradin, Aschero, Aguerre 1984/85), hacia mediados de los '70 mostraba su dificultad para relacionar datos del campo e inferencias analíticas de orden difusionista.

\section{La "publicación oral" de la Tipología.}

La primera transmisión nacional de la Tipología, ya puesta

proyectil o una raedera entran en esa categoría pero también quedan incluidas una lasca de descarte o un núcleo abandonado (Primera convención 1964:17)

25 Nació en 1911 en París. Se doctoró en Letras y en ciencias, fue profesor de Prehistoria y Etnología. M. Mauss, dirigió su tesis doctoral sobre la Arqueología del Pacífico Norte. Desde 1933, trabajó en los departamentos de Etnología del Museo Británico (Londres) y el Museo del Hombre (París). En 1964, creó el concepto de Cadena Operativa (chaîne opératoire), un conjunto de pasos encadenados que conforman a su juicio la producción de artefactos líticos, desde la recolección de materia prima, pasando por las fases de fabricación, utilización, reconstrucción (afilado, reavivado, reaprovechamiento.) y reutilización. Falleció en París el 19 de febrero de 1986. a prueba por su autor en el Seminario de 1977, tuvo lugar en el espacio mayor del V Congreso Nacional de Arqueología dónde Aschero compartió la presentación del trabajo de los siete sitios con sus alumnos de la "Sala de las columnas". Este encuentro fue el primer espacio institucional y académico a escala nacional fuera de la Facultad porteña, donde Aschero pudo interactuar con reconocidos arqueólogos de otras regiones y mostrar su producción y su estilo de trabajo ya como un par. Las dos últimas jornadas del Congreso se centraron en las investigaciones vinculadas a la temática de los cazadoresrecolectores de las regiones de Pampa y Patagonia, y en su tramo final se presentaron las ponencias correspondientes al Arte Rupestre de Patagonia. Las Actas del Congreso con los trabajos sobre la agricultura del NOA y Cuyo conformaron el Tomo I y fueron publicadas; en tanto el Tomo II, con las investigaciones sobre Pampa y Patagonia y el Arte Rupestre patagónico, no llegó a ver la luz. De todos modos el desafío estaba planteado y la presentación inédita de San Juan no hizo sino fortalecer la actividad grupal que había propuesto Aschero con su Seminario y que, excediendo los propios límites de un curso universitario, puso en marcha entre sus estudiantes la necesidad de dar continuidad a una forma de trabajo grupal con preponderancia a la especialización y con una marcada acentuación analítica no contemplada en la enseñanza oficial de la licenciatura porteña. Así pues ello terminó redundando en la conformación de diferentes equipos de investigación que a partir de los distintos intereses individuales de sus miembros fueron delineando grupos de trabajo en todo el país abarcando temas como la cestería prehistórica, la textilería prehistórica, la etnohistoria, zoo-arqueología o arqueofauna, la paleobotánica, y las dos manifestaciones de arte prehistórico, el rupestre y el mobiliar. Ese fortalecimiento terminó de concretarse en una nueva modalidad de reunión con la creación del Centro de Investigaciones Arqueológicas (CIA), agrupación extra-académica creada tanto por algunos de los estudiantes de arqueología porteños como por graduados recientes que sufrían una desvinculación "forzada" de la arqueología al finalizar su formación de grado. Esta agrupación, que funcionó entre 1978 y 1981, vino a paliar las necesidades de desarrollo que no ofrecidas por la formación oficial de la carrera aún regida por lineamientos histórico-cultural. No era éste sólo un espacio extra-universitario sino, más bien, un complemento en el camino del crecimiento profesional, que contaba con una firme participación de sus miembros mediante encuentros de trabajo y discusiones sobre cuestiones de práctica de la arqueología, en el ámbito de sus propios hogares y que contaba con el asesoramiento de Aschero.

\section{El seminario en campaña. El viaje a "Cerro de los indios".}

Aún en circunstancias en que el imperio del PRN imponía la represión de toda actividad política durante la segunda mitad de los '70, la secuencia iniciada con el hilo 
conductor de la Tipología de Carlos y puesta en juego a partir del Seminario de la "Sala de las columnas" y la posterior presentación en el V Congreso Nacional, tuvo su continuidad en emprendimientos que prolongaron esta suerte de pasaje hacia otra instancia y que nos permite describir de manera simbólica el camino de cambio iniciado por Aschero y su grupo de estudiantes y graduados recientes, en plena dictadura y bajo el sesgo institucional de los herederos de Menghin.

Uno de esos emprendimientos fue el de la campaña arqueológica a "Cerro de los Indios" en la altiplanicie de la provincia de Santa Cruz realizada en 1977. En tiempos en que llevar alumnos al campo era arriesgar la vida, Aschero trasladó a estudiantes de ambos sexos a una campaña arqueológica que fue calificada por las autoridades de la carrera como "subversiva"26. A lo largo de un mes, el equipo integrado por algunos de los "iniciados" que habían integrado el Seminario, como Eduardo Crivelli, Guillermo Mengoni, María José Figuerero Torres, Carlos López, Alfredo Fisher, Andrés Rodríguez Larreta, Ana María Llamazares, Mónica Solís, María Onetto, Cristina Bellelli y otros que ya habían obtenido su título de grado como María Teresa Boschín y Lidia Nacuzzi, formó parte de una experiencia en el espacio patagónico que identificaron con el llevado a cabo por Leroi-Gourhan en Francia:

"Fuimos divididos por tandas, y fue el primer intento estratigráfico de trabajo de planta. Carlos intentó lo de Pincevant ${ }^{27}$, trabajando con pisos de ocupación, buscando a la gente que había estado allí en el pasado, mirando el funcionamiento de su espacio. Se intentaba vincular todo, reconociendo la importancia del espacio, integrando el paisaje" (Entrevista a C. Bellelli 01/06/2006).

Esta nueva experiencia desarrollaba una mecánica de trabajo que Aschero ensayaba por primera vez en la arqueología nacional. Según la evocación de Bellelli, "Cerro de los Indios" "fue operar en un sitio nacional y propio con un enfoque que Leroi había aplicado en Francia", valiéndose de la técnica de décapage. Con la organización de esta campaña, Aschero desafiaba no sólo las restricciones nacionales a la reunión de más de dos personas en sitios públicos, sino también las de la autoridad de la licenciatura. A su regreso Aschero fue reconvenido por Bórmida quien le planteó su total desacuerdo por llevar a una campaña arqueológica a

\footnotetext{
26 Para un relato pormenorizado de esta y otras "apreciaciones" de índole política vertidas en la época ver Luco 2009:91 y ss.

27

excavación del asentamiento magdaleniense de Pincevent (desde 1964), Leroi-Gourhan renovó la metodología de excavación gracias a la conservación del sitio. Desarrolló la excavación en extensión, a través del método de décapage que consistía en una excavación en horizontal, siguiendo la topografía de la capa arqueológica, observando la estratigrafía y anotando todos los detalles a ritmo muy lento, dejando al descubierto amplias zonas de un mismo estrato arqueológico, desplegando un análisis espacial de este hábitat prehistórico.
}

gente de "inconveniente" posición política (Entrevista a Aschero 13/07/2007).

La continuidad de esta suerte de pasaje simbólico con el que pretendemos demostrar que en un principio el cambio de paradigma tomó características de índole metodológica, se concretó con dos emprendimientos finales: la concreción de una nueva asignatura en el espacio institucional universitario de la UBA (1979) y la convocatoria a las "Primera Jornadas de Tecnología y Tipología Lítica" (1980). La asignatura "Ergología y Tecnología" significó una innovación didáctica para la licenciatura porteña ya que fue la primera incorporación al conjunto de materias regulares de la especialidad desde la constitución de la carrera de grado en 1958. Tanto su denominación como su temática fueron decididas por sus dos mentores, Aschero y Aguerre, ganando un nuevo espacio de interlocución para estudiantes avanzados y egresados recientes de la especialidad arqueológica. Su dictado, de duración anual, data de 1979 unos meses después de la muerte de Bórmida en septiembre de 1978 quien fuera el que la encomendara y autorizara ${ }^{28}$. En su dictado se incluían las transformaciones metodológicas que Aschero venía introduciendo desde el Seminario, las reuniones del CIA y la campaña a Cerro de los Indios. Pero "Ergología" fue además un espacio donde se conocía y discutía la aplicación de la New Archaeology ${ }^{29}$ el nuevo paradigma proveniente de la academia anglosajona. Tal fue el escenario académico que encontró otro actor de la práctica arqueológica patagónica practicada desde la UBA, Luis Borrero (n 1947) para afirmar en términos teóricos, el ingreso de esta nueva perspectiva.

"Ergología y Tecnología" fue una instancia institucional que se vivió como "ganada" desde adentro de la licenciatura porteña y desde dónde se tendió un nexo

28 Una breve narración de Aguerre de 1988 sirve para rescatar el sentido acerca de las condiciones en que se produjo esta creación: “Ergología comienza en 1979, una experiencia que... quiero aclarar cómo se gestó esa cátedra. Una tarde llegó Bórmida al Instituto, empezamos a hablar... Y él nos dijo:-¿Qué materia les parece que hace falta en la orientación? Para qué!...Nos empezamos a despachar...hace falta algo que permita al alumno poder recapacitar sobre todos los conocimientos que está recibiendo, que no puede elaborar, que no puede discutir que nos están llegando trabajos, que realmente por falta de tiempo, continuamente eran dejados de lado" (Aguerre en JCGCA 1988: 115).

29 La revolución epistemológica de la New Archaeology se originó en la década de 1960 pero ingresó a la Argentina en la década siguiente, expresando una creciente necesidad de revisión crítica de los conceptos y las nociones en uso. Las nuevas argumentaciones reordenaron las categorías (atributos, artefactos, culturas y grupos culturales) empleadas por los arqueólogos contemporáneos y se cristalizaron en los trabajos de los anglosajones Lewis Binford (1962 y 1965) de Estados Unidos y David Clarke (1968) de Inglaterra, artífices de dicho cambio paradigmático. Este nuevo discurso acerca del pasado abogaba por un status científico que les permitiría a los investigadores enunciar hipótesis y modelos contrastables científicamente, suplantando las interpretaciones históricas y altamente especulativas de las escuelas precedentes. 
con aquellos colegas cuyas ausencias resentían tanto por exilios externos como internos-tales por caso los de Orquera y Lafón-como con una joven generación descreída de la Arqueología "oficial", que intentaba subsanar su exclusión con las iniciativas extra-universitarias de nucleamiento (Luco 2009: 95). En tanto, las "Primeras Jornadas de Tecnología y Tipología Líticas" convocadas por el CIA para el fin de semana del 24 al 26 de octubre de 1980 en la sala de reuniones de la sucursal Colegiales del Banco de Crédito Cooperativo (Credicoop), esto es, en un espacio externo a la jurisdicción universitaria oficial tenían el objetivo de discutir "las principales líneas de trabajo en el tema (tipológico) para las regiones de Pampa, Patagonia y Tierra del Fuego"(PJT 1980:3). Así, la moderna sala de conferencias del Banco Credicoop albergó a un poco más de un centenar de especialistas. Los fundadores del CIA y organizadores ${ }^{30}$ de las Jornadas fueron anfitriones no sólo de los arqueólogos pertenecientes a las universidades nacionales en las que se dictaba la Licenciatura específica- la de Ciencias Antropológicas de la UBA y la de Ciencias Naturales de la UNLP- sino también de quienes se habían formado en universidades donde la arqueología se impartía como especialización de la carrera de Historia, como era el caso de la Universidad del Litoral en Rosario y de las sedes Mendoza y San Juan de la Universidad de Cuyo. Con el fin de lograr una clasificación uniforme para todo el país a lo largo de tres días se ofrecieron distintas instancias de encuentro reservadas a la discusión de temáticas como: el marco teórico para el encuadre técnico-tipológico, la conceptualización de términos técnicos en uso profesional tales como artefacto, instrumento y utensilio, aspectos tecnológicos de la talla, retalla y retoque, definiciones y nomenclatura de piso, contexto e industria, así como la definición y clasificación de los tipos de sitios donde se localizaban habitualmente los hallazgos líticos ${ }^{31}$ (Luco 2009:43).

Los organizadores de las Jornadas habían dispuesto una dinámica de trabajo que consistía en una presentación a cargo de un especialista para cada región que, en un métier tan reducido, era ya conocido por todos. Cada uno de ellos expuso así la experiencia obtenida con su propia tipología concebida a partir de las necesidades impuestas por sus investigaciones. El intercambio de experiencias

\footnotetext{
30 La organización y coordinación de las Jornadas estaba a cargo de Ana María Llamazares, Cristina Vulcano y María Teresa Boschín, egresadas en 1976 de la especialización arqueológica de la licenciatura porteña.

${ }^{31}$ Una de las novedades del encuentro fue la inclusión de un "Taller de talla" para la práctica de la "arqueología experimental" que, implementada en los Estados Unidos de Norteamérica en campamentos al aire libre entre 1970 y 1980, posibilitaba que el arqueólogo, situado como protagonista del proceso tecnológico, investigara por sí mismo las técnicas de confección de las herramientas prehistóricas. Nora Flegenheimer y Hugo Nami, dos estudiantes avanzados de La Plata y Buenos Aires respectivamente que en distintos momentos de su formación viajaron a Estados Unidos para realizar el aprendizaje de esta réplica de las tallas prehistóricas tutelaron, en las Jornadas Líticas, las prácticas en este taller.
}

y de interpretaciones se apoyó en la presentación de los trabajos de Austral, Ceballos y Orquera entre otros. La presentación de la arqueología de la Patagonia continental estaba a cargo de Carlos Aschero, pero ante su ausencia, fue expuesta por Orquera. Probablemente el hecho de haber elegido un escenario como el de la sede de una organización cooperativa bancaria creada en 1979 sobre el principio solidario de la ayuda mutua y, por ende, ajeno a la jurisdicción del Instituto de Antropología de la Universidad de Buenos Aires y a un clima general de persecución ideológica produjo como consecuencia que, quienes revistaban en la UBA se vieran envueltos en dilemas de lealtades contrapuestas. La ausencia de Aschero, ya parte del plantel docente de la facultad, quien se excusó a través de una carta que fue leída a la concurrencia la tarde de la apertura, se interpretó como respuesta a las presiones de su superior jerárquico en el Instituto de Antropología de la UBA (Luco 2009:44).

Las "Primeras Jornadas de Tecnología y Tipología líticas" encarnaron la concreción de las nuevas maneras de operar en el campo arqueológico de Patagonia, aquellas que, iniciadas con la propuesta tipológica de Aschero junto a las otras iniciativas nacionales ya mencionadas, procuraron romper una continuidad no sólo ecológica y prehistórica, de Patagonia, sino también la teórica y académica, la del difusionismo de la "Escuela de Buenos Aires".

\section{Conclusiones}

Hasta aquí hemos intentado exponer, mediante una reflexión fundada en un análisis etnográfico, por qué el caso de la arqueología patagónica practicada desde la licenciatura antropológica de Buenos Aires, desmiente que los contextos represivos imperantes en los últimos regímenes de facto, se hayan traducido en períodos de retracción, conservadorismo e inmovilidad en el mundo académico. A través de la presentación de una serie de hitos, a nuestro juicio significativos, creemos haber demostrado que a partir de iniciativas autónomas que nacieron de un grupo de estudiantes, egresados recientes y jóvenes docentes, se inició y llevó a cabo un cambio de paradigma en la enseñanza y práctica de la arqueología patagónica practicada desde la UBA en el período 1975-1983, y que el mismo se desarrolló en dos instancias. Al postular que este cambio se inició en una instancia metodológica a la que le siguió un cambio teórico, estoy señalando que es posible pensar en los procesos de cambio de paradigma no como trayectorias que observan una sucesión universal de etapas-al modo evolucionista-sino más bien como procesos locales. También señalo que su génesis operó desde el interior de la institución universitaria que albergaba a sus hacedores pero que aún conducía la teoría oficial de la "Escuela de Buenos Aires".

Dicho esto, debiera quedar claro que no suponemos 
que la voluntad creativa de estos arqueólogos pudiera plasmarse de igual modo y con la misma trascendencia que en un contexto cooperativo y tolerante al disenso. Quizás una clave de cuánto incidió el modelo de pensamiento único que reinaba en la sociedad argentina de entonces, pueda encontrarse en el hecho, naturalizado por la comunidad arqueológica, de que una obra tan influyente como la "Tipología de Carlos" se mantuvo inédita aún cuando fue una de, si no la más utilizada y aplicada por "los líticos". Igual destino que el trabajo de los siete sitios producto del seminario. ¿Acaso jugó allí la voluntad de su autor y/o la de las autoridades de entonces? Probablemente su condición de texto inédito mantuvo a ambas obras, aunque sólo sea formalmente, fuera de la arqueología oficial. Quizás, su circulación por carriles "informales", entendiendo por ello el que no estuviera incluido en las cátedras y que la proveyeran Aguerre y Aschero en sus clases, permitió consolidar las redes entabladas por personas entre las cuales primaba la confianza de querer ser socializadas en ellas. Estamos afirmando, a modo de hipótesis, que su condición de inéditas asociada a lo intersticial y liminal ${ }^{32}$ que caracterizó a la Tipología, permitió el tránsito hacia el cambio, aunque extendiendo su carácter informal o no decididamente institucionalizado, a toda su comunidad de usuarios y desde ya a su propio autor. Pero vayamos paso a paso.

El ingreso de Aschero al escenario de la práctica profesional porteña aceleró el "quiebre" definitivo de la autoridad académica de la escuela histórico-cultural, al convalidar en el campo la pérdida de efectividad de los instrumentos teóricos del viejo paradigma. La nueva dirección que la arqueología procesual o New Archaeology le imprimía a la práctica mundial de la disciplina ganó adeptos también en el ámbito profesional local. Y en esta instancia de cambio fue también el lugar intersticial, liminal o transicional que ocupó Aschero tanto en la interacción con colegas y discípulos como en las nuevas instancias teóricas, el que posibilitó la articulación hacia el cambio.

En principio y frente a una sociedad con un sistema estructurado, diferenciado y a menudo jerárquico, de posiciones político-jurídicas-económicas con múltiples criterios de evaluación desde el punto de vista de subordinación, ordenamiento y control, representado por la organización institucional instaurada por los históricoculturales, hemos podido reconocer el surgimiento de lo que llamamos período o instancia liminal. $Y$ al caracterizarlo así, mediante la descripción de una suerte de pasaje por diversos hitos, hemos distinguido una communitas integrada por Aschero, los estudiantes y los egresados recientes ubicada por fuera de la instancia oficial que se expresó en manifestaciones tales como las

\footnotetext{
32 Se utiliza aquí esta noción de liminalidad en el sentido planteado inicialmente por van Gennep (1960), en su trabajo sobre Ritos de pasaje y más tarde por Victor Turner (1969). Así el período liminal o intermedio debe entenderse como con un sentido de ambigüedad en tanto atraviesa un entorno cultural con pocos o ninguno de los atributos del estado pasado o del venidero.
}

del CIA, la Sala de las Columnas, la Tipología inédita, el trabajo de los siete sitios, la campaña de Cerro de los Indios y la conquista de una nueva asignatura. Fue ésta sociedad "sin estructura" y de iguales la que instituyó una estructura social nueva aunque fragmentada, cuya organización social académica se sustentó sobre la "Tipología de Carlos".

De la misma manera que Aschero ensambló la tipología francesa con la estadounidense para clasificar e interpretar los materiales locales, operó como artífice de la articulación entre su propia modificación metodológica, originada desde el espacio institucional que lo formó en arqueología prehistórica histórico-cultural, y las tareas de los graduados recientes deseosos de abrevar en un nuevo paradigma. Así, los atributos profesionales e institucionales de Aschero, tal como si fueran los atributos descriptos por él mismo para una herramienta lítica, lo ubican necesariamente junto a su práctica en aquella época, en un territorio liminar o ambiguo que hace que tanto su persona como sus acciones escapen tenazmente a todo sistema clasificatorio. La combinación de sus condiciones personales y su autoridad científica fortaleció su figura habilitándolo para mediar en una disputa en el ámbito disciplinar argentino transformando, desde entonces, la perspectiva arqueológica sobre Patagonia.

El orden aparentemente cronológico con que me he referido a este proceso de cambio en el que destaco una precedencia del aporte metodológico y técnico de Aschero, aprovechando los intersticios de la academia oficial con respecto al cambio paradigmático que introdujo Borrero, es una construcción nuestra que reproduce el orden que mis colegas-informantes lograron transmitirme, y constituye, a nuestro juicio, un modo posible en que estos protagonistas de los acontecimientos aquí narrados pudieron llevar a cabo modificaciones de cara al orden histórico-cultural establecido.

Al igual que la relación de tensión entre política y academia aludida en el título de este artículo, fue también de tensión el vínculo que la arqueología lítica porteña mantuvo con las manifestaciones de la política nacional precisamente porque no se fundieron en una misma cosa. Dicha tensión estuvo acrecentada por la ineludible contemporaneidad del crecimiento y desempeño académico arqueológico en la UBA con el PRN y sobrellevada desde el compromiso con la arqueología por Aschero y más tarde por Borrero.

Para finalizar deseo hacer dos salvedades. Por un lado, advertir que se trata aquí de la presentación de una etnografía antropológica de una comunidad académica de la cual yo misma egresé. Es cierto que trabajar con colegas sobre esos mismos colegas, esto es, investigarlos, es una empresa riesgosa. Se juegan aquí no sólo las capacidades analíticas propias de quien esto escribe, sino también la pretensión lógica de reconocimiento de tantos (en este caso) arqueólogos, algunos sumamente 
destacados, que han contribuido desde sus clases y sus artículos, a veces olvidados, a que la arqueología argentina y la antropología en general, pudieran salir de su provincianismo sureño para ingresar al mundo de las ideas académicas. En todo caso, este artículo debiera ser leído como la ponderación del enorme potencial de nuestros intelectuales arqueólogos, potencial que no pudo ser coartado pese a lo "irrespirable" que se tornó el período en estudio. Por otro lado, la segunda salvedad es recalcar que la centralidad otorgada en mi narración a quienes encabezaron el cambio, no pretende atribuirles una prioridad extra-humana, al estilo acostumbrado de las biografías de los próceres de cada disciplina, sino más bien mostrar figuras académicas que construyeron parte de su desarrollo junto a colegas, profesores, directores, inspiradores, estudiantes y amigos, durante la época que les tocó transitar.

Buenos Aires, 18 de agosto de 2010.

\section{Agradecimientos}

Este artículo ha sido elaborado como parte del PICT Redes 1728 (2006) "Antropología social e histórica del campo antropológico en la Argentina, 1940-1980" de la ANPCyT.

\section{Bibliografía}

Aschero, C 1975a. Ensayo para una clasificación morfológica de artefactos líticos aplicada a estudios tipológicos comparativos. 162 páginas. Inédito. Buenos Aires.

Aschero, C 1975b. Secuencia arqueológica del Alero de las Manos Pintadas (Las Pulgas, departamento Río Senguer, Chubut). Relaciones de la Sociedad Argentina de Antropología, tomo IX: 187-209. Buenos Aires.

Aschero, C 1983. Ensayo para una clasificación morfológica de artefactos líticos. Apéndice A-C (Revisión) 80 Págs. Buenos Aires.

Aschero, C 1987. Tradiciones culturales en la Patagonia central. Una perspectiva arqueológica. Primeras Jornadas de Arqueología de Patagonia: 17-26. Rawson, Argentina, Gobierno de la Provincia de Chubut.

Aschero, C 2000. El poblamiento del territorio. Myriam N. Tarragó. Los pueblos originarios y la conquista, pp. 17-60. Buenos Aires, Editorial Sudamericana.

Austral, A. 1966. La talla por percusión. Etnía N 3: 9-20. Olavarría, Provincia de Buenos Aires, Museo Municipal "Dámaso Arce".

Bartolomé, .L 1980 La Antropología en Argentina: Problemas y Perspectivas América Indígena XL (2): 207215.
Bonnin, M. y A. Laguens 1984-1985. Acerca de la arqueología argentina de los últimos 20 años a través de las citas bibliográficas en las revistas Relaciones y Anales de Arqueología y Etnología. Relaciones de la Sociedad Argentina de Antropología XVI (Nueva Serie): 7-25. Buenos Aires.

Boschín M. T. y A. M Llamazares 1980. (Compils.) Primeras Jornadas de tecnología y tipología líticas. (Buenos Aires, 24, 25 y 26 de octubre de 1980). Centro de Investigaciones Antropológicas. (CIA) Buenos Aires, s.f, 68 págs.

Boschín M. T. y A. M Llamazares 1984 La Escuela Histórico-Cultural como factor retardatario del desarrollo científico de la arqueología argentina. Etnía 32: 101-156. Olavarría, Provincia de Buenos Aires.

Boschín M. T. 1993. Historia de las investigaciones arqueológicas en Pampa y Patagonia. En: Runa, Tomo XX: 111-144(1991-1992).

CGAJA. Colegio de Graduados en Ciencias Antropológicas 1989. Jornadas de Antropología: 30 años de la carrera en Buenos Aires (1958-1988). Universidad de Buenos Aires, Facultad de Filosofía y Letras.

Centro Argentino de Etnología Americana. 1985. Antropología. Evolución de las Ciencias en la República Argentina. 1872-1972" Tomo X. Sociedad Científica Argentina.

Fernández, J. 1982. Historia de la Arqueología Argentina. Mendoza, Asociación Cuyana de Antropología.

Fígoli, L. 1995. A Antropología na argentina e a construcao da nacao. En Roberto Cardoso de Oliveira y Guilhermo Ruben (orgs.). En: Estilos de Antropología. Campinas, Unicamp.

Garbulsky, E. 1987. José Imbelloni, positivismo, organicismo y racismo. Cuadernos 3/87. Escuela de Antropología de Rosario.

Gradin, C, C Aschero y A. M Aguerre 1977. Investigaciones arqueológicas en la Cueva de las Manos, Estancia alto río Pinturas (Alto Río Pinturas, Santa Cruz). Relaciones de la Sociedad Argentina de Antropología, X:183-227. Buenos Aires.

Gradín, C, C Aschero y A M Aguerre 1979. Arqueología del Área Río Pinturas. Relaciones de la Sociedad Argentina de Antropología 14:177-194, Buenos Aires.

González, A. R. 1985. Cincuenta años de arqueología el noroeste argentino (1930-1980). Apuntes de un casi testigo y algo de protagonista. American Antiquity 50(3). 
González, A. R. 1991-1992. A cuatro décadas del comienzo de una etapa. Apuntes marginales para la historia de la Antropología Argentina. Runa XX: 91-110. Buenos Aires. ICA. FFyL.

González, A. R. 2000. Tiestos dispersos. Voluntad y azar en la vida de un arqueólogo. Buenos Aires, Editorial Emecé.

Guber, R y S Visacovsky 1999. Controversias filiales: la imposibilidad genealógica de la Antropología Social de Buenos Aires. Relaciones de la Sociedad Argentina de Antropología. XXII-XXIII:25-53.

Guber, R y S Visacovsky 2000. La Antropología Social en la Argentina de los '60 y '70. Nación, marginalidad crítica y el "otro" interno. Desarrollo Económico, 40(158).

Guber, R 2003: Esther Hermitte y los orígenes de la Antropología Social de Buenos Aires. Manuscrito.

Guber, R 2006. Linajes ocultos en los orígenes de la antropología social de Buenos Aires. Avá. Revista del Postgrado en Antropología Social de la Universidad Nacional de Misiones, Argentina 8:26-56.

Guber, R 2006. Crisis de presencia, universidad y política en el nacimiento de la antropología social porteña. Revista Colombiana de Antropología 43:263-298.

Guber, R, M Bonnín, y A Laguens 2008. Tejedoras, topos y partisanos Nociones y prácticas del trabajo de campo en la antropología argentina. Relaciones de la Sociedad Argentina de Antropología. Volumen homenaje a los 70 años de su fundación.

Gurevich, E y E. M. Smolensky 1987. La Antropología en la Universidad de Buenos Aires, 1973-1983. Informe PIA. CONICET. Mimeo.

Haber, A 1994. Supuestos teórico-metodológicos de la etapa formativa de la arqueología de Catamarca (18751900).: Publicaciones Arqueología 47: 31-54. Córdoba: CIFFyH, UNC.

Herrán, C. 1987. Antropología Social en la Argentina. Apuntes y perspectivas En: Simposio sobre Teoría e Investigación de la Antropología Social Mexicana. El Colegio de México. Mayo 11-14.

Imbelloni, J. 1949. Informe preliminar sobre la expedición a la Patagonia. Elevado al Delegado Interventor de la Facultad de Filosofía y Letras, Dr. Enrique Francois, y al Administrador General de Parques Nacionales y Turismo, Ten. Coronel Napoleón A. Hirsuta.

Imbelloni 1931. Introducción al estudio de las civilizaciones según el método histórico-cultural. Buenos
Aires, Imprenta de la Universidad de Buenos Aires.

Kohl, P. L y J. A Pérez Gollán, 2002. Mixing religión, politics, and prehistory: the life and writings of $O$. Menghin. Current Anthropology. 43:561-586.

Lázzari, A 2004. Antropología en el Estado: el Instituto Étnico Nacional (1946-1955) Comps. Federico Neiburg y Mariano Plotkin En: Intelectuales y expertos. La constitución del conocimiento social en la Argentina. 203-219 Buenos Aires, Paidós.

Luco, S 2009 De prehistoriadores a arqueólogos. Una etnografía del cambio de paradigma en la práctica académica de la arqueología patagónica. UBA 19751983. Tesis de Maestría aprobada en IDES/IDAES/ UNSAM. Ms.Madrazo, G. 1985. Determinantes y orientaciones en la Antropología argentina. Boletín del Instituto Interdisciplinario Tilcara, 1:13-56. Instituto Interdisciplinario Tilcara Buenos Aires, Universidad de Buenos Aires. Facultad de Filosofía y Letras.

Márquez Miranda, F. 1941. Fritz Graebner y el método etnológico. Notas del Museo de la Plata. Tomo VI. Antropología N ${ }^{\circ} 22$. La Plata, Provincia de Buenos Aires.

Menghin, O. 1952a Las pinturas rupestres de Patagonia. En: Runa V (1.2), Buenos Aires.

Menghin, O. 1952b. Fundamentos cronológicos de la prehistoria de Patagonia. En: Runa 5: 23-43, Buenos Aires.

Menghin, O. 1957. Estilos del arte rupestre de Patagonia. En: Acta Praehistórica, I, Buenos Aires.

Menghin, O..1963. Industrias de morfología protolítca en Suramérica. En Anales de la Universidad del Norte, $N^{\circ} 2$ : 69-77. Antofagasta. 1963.

Nuñez Regueiro, V. 1972. Conceptos teóricos que han obstaculizado el desarrollo de la arqueología en Sud América. En: Estudios Arqueológicos, 1. Cachi, Salta.

Primeras Jornadas de Tipología y Tecnología Líticas.(PJT). Buenos Aires. 1980.

Pérez de Micou, C 2006. El modo de hacer las cosas. Artefactos y ecofactos en Arqueología. ICA. Facultad de Filosofía y Letras. UBA.

Podgorny, I 2004. Antigüedades incontroladas. La arqueología en la Argentina, 1910-1940. Comps. Federico Neiburg y Mariano Plotkin. En: Intelectuales y expertos. La constitución del conocimiento social en la Argentina. 147-174. Paidós, Buenos Aires.

Politis, G 1988. Paradigmas, modelos y métodos en la Arqueología de la Pampa bonaerense. En: Arqueología 
contemporánea argentina. Ediciones Búsqueda, Buenos Aires.

Politis, G 1992 Política nacional, arqueología y universidad en Argentina. Politis (ed.). En: Arqueología de América Latina hoy. Biblioteca Banco popular, Bogotá, Colombia, pp 70-87.

Politis, G 2004 Theoretical Lanscape and the methodological development of archaeology in Latin America. En: American Antiquity 68 (2): 245.272

Ratier, H. E y R. Ringuelet, 1997. La Antropología Social en la Argentina: un producto de la democracia. Horizontes antropológicos 3(7):10-23.

Robben. A. 1999 The Fear of Indifference: Combatants' Anxieties about the Political Identity of Civilians during Argentina's Dirty War. En Kees Koonings y Dirk Kruijt (eds.), Societies of Fear: The Legacy of Civil War, Violence and Terror in Latin America, 125-140.London. Zed Books.

Sanguinetti, A. C. 1972. Introducción y apéndice a Menghin traducción aparecida Anales de Arqueología y Etnología, tomo XXVI.
Sanguinetti A. C y M. L Schlegel 1972. Industrias arcaicas del río Neuquén. Relaciones de la Sociedad Argentina de Antropología, tomo VI: 91-108. Buenos Aires.

Tarragó, M. 2003. La arqueología de los Valles Calchaquíes en perspectiva histórica. Anales Nueva época. Local, regional, global: prehistoria, etnohistoria en los Valles calchaquíes. Goteborg Universitet.

Turner, V. 1969 Liminality and communitas. En: The ritual process. Structure and anti-structure. Chicago, Aldine Publishing Company.

Visacovsky, S E, R Guber y E. Gurevich 1997. Modernidad y tradición en el origen de la carrera de Ciencias Antropológicas de la Universidad de Buenos Aires. En: Redes 10(4):213-258.

Visacovsky, S. E. 2002 El Lanús. Memoria y Política en la construcción de una tradición psiquiátrica y psicoanalítica argentina. Buenos Aires, Alianza Editorial.

Visacovsky, S. E. 2007 Cuando las sociedades conciben el pasado como "memoria": Un análisis sobre verdad histórica, justicia y prácticas sociales de narración a partir de un caso argentino. En: Antípoda 4:49-74. 